\title{
Cuboid Morphology of a Basal Anthropoid from the Eocene of China
}

Daniel L. Gebo ${ }^{a}$, Marian Dagosto ${ }^{b}$ K. Christopher Beard ${ }^{c, d}$, Xijun Ni ${ }^{\text {e }}$

a Department of Anthropology, Northern Illinois University, DeKalb, IL 60115, United States

${ }^{\mathrm{b}}$ Department of Cell and Molecular Biology, Feinberg School of Medicine, Northwestern University, Chicago, IL 60611, United States

${ }^{c}$ Biodiversity Institute, University of Kansas, Lawrence, KS 66045, United States

${ }^{d}$ Department of Ecology \& Evolutionary Biology, University of Kansas, Lawrence, KS 66045, United States

${ }^{\mathrm{e}}$ Institute of Vertebrate Paleontology and Paleoanthropology, Chinese Academy of Sciences, Post Office Box 643, Beijing 100044, China

Key Words: Eosimiidae

A small primate cuboid (IVVP V 12319; Fig. 1) has been recovered from Fissure $D$ at the middle Eocene Shanghuang fissure-fillings in southern Jiangsu Province, China (Beard et al., 1994). Its length (3.6 $\mathrm{mm}$ in total length), width $(2.4 \mathrm{~mm}$ at the calcaneocuboid joint; distal width $1.75 \mathrm{~mm})$ and height $(1.5 \mathrm{~mm})$ resemble the dimensions in extant mouse lemurs, indicating that the new cuboid pertains to a smallbodied primate with an adult body mass of $30-60 \mathrm{~g}$. As such, this cuboid is too small to be allocated to any of the known adapiform taxa at Shanghuang, all of which are estimated to have an adult body mass $>200 \mathrm{~g}$, leaving its allocation to one of the small 
haplorhine groups that are documented from this site (Gebo et al., 2012). At least four groups of haplorhines are known from the Shanghuang fissure-fillings, including omomyids (represented by the large-bodied taxon Macrotarsius macrorhysis), tarsiids (represented by Tarsius eocaenus), eosimiids (represented by Eosimias sinensis and probably other unnamed taxa), and a group of relatively advanced anthropoids that remain poorly documented (Beard et al., 1994; Gebo et al., 2012). IVPP V 12319 is far too small to be referred to Macrotarsius macrorhysis (the adult body mass of which is estimated to be > $900 \mathrm{~g}$; see MacPhee et al., 1995), which is the only omomyid currently described from Shanghuang. Nevertheless, a number of small postcranial elements are known from Shanghuang that generally resemble those of the Omomyidae (Gebo et al., 2012), suggesting that either additional omomyid taxa are present at Shanghuang or another group of a relatively basal haplorhine with an omomyid-like postcranial anatomy occurred there. In either case, IVPP V 12319 is unlikely to pertain to an omomyid-like postcranial group as omomyid cuboids are relatively elongated and display a narrow distal region with a narrow joint surface relative to the proximal joint surface (Szalay, 1976; Fig. 2, left), in marked contrast to IVPP V 12319 (Fig. 2, middle). IVPP V 12319 is dissimilar to tarsiid cuboids as these cuboids possess a flat proximal joint surface without a distinct cuboid process and have a widely laterally flaring proximal region in dorsal view (Gebo, 1987). IVVP V 12319 possesses a distinct cuboid process and lacks the distinctive cuboid features known for Tarsiidae, suggesting that IVVP V 12319 must be allocated to one of the two anthropoid groups known from Shanghuang. The more advanced anthropoid group at Shanghaung is extremely rare, and our working hypothesis is that the IVPP V 12319 
cuboid should be allocated to the basal anthropoid group Eosimiidae, which is the most abundant primate taxon documented from the Shanghuang fissure-fillings.

In terms of morphology, the IVPP V 12319 cuboid is a rectangular shaped bone with a strong lateral groove for the peroneus longus tendon (Fig. 1). It differs from the cuboids of other common fossil mammals at Shanghuang such as rodents (see Rose et al., 2004) or lagomorphs (see Meng et al., 2009; or Fostowicz-Frelik et al., 2015) as both these taxa exhibit cuboids with very prominent plantar tuberosities. Further, rodents exhibit proximal and distal cuboid joint surfaces that are non-parallel in their articulations while lagomorph cuboids also show a projecting cuboid-ectocuneiform facet. None of these cuboid features characterize IVPP V 12319.

Other mammalian taxa at Shanghaung, including carnivores, artiodactyls, and perissodactyls, are much larger than the species to which the IVPP V 12319 cuboid belonged. Similarly, most of the "insectivores" at Shanghuang are tiny and insectivore cuboids are more compact with non-parallel proximal and distal joint surfaces (see Dunn and Rasmussen, 2009). Insectivores such as tenrecs exhibit longer cuboid shapes that are pinched in the middle, a morphological feature not seen in IVPP V 12319 (see Salton and Szalay, 2004). Penkrot et al. (2009) describe and illustrate cuboids of the small condylarths Apheliscus and Haplomylus. Although these cuboids are elongate, like IVPP V 12319, they lack the key primate features of the cuboid pivot and the deep groove for the peroneus longus muscle tendon. They also differ from the Shanghuang cuboid in having a mediolateral constriction and a facet for the talus. Extant tree shrews exhibit cuboid shapes more similar to the Omomyidae with a 
shortened distal joint surface (see Sargis, 2002), a feature that does not characterize IVPP V 12319.

The cuboid pivot on IVPP $\vee 12319$, a distinctive primate feature associated with foot inversion during grasping (Decker and Szalay, 1974), is centrally located along the calcaneocuboid joint surface, and, as noted above, both the proximal and distal joint surfaces are wider than they are tall. The medial side of the cuboid bears a small distal facet and two additional facets located midway along the proximodistal length of the bone for the ectocuneiform. The lateral side shows a prominent groove for the peroneus longus tendon. The plantar surface exhibits a strong mediolaterally and obliquely oriented bony ridge representing the proximal edge for the peroneus longus tendon groove. Proximal to this edge lies a roughened and flatter surface of the cuboid where substrate contact is made during semiplantigrade foot touchdown (Gebo, 1992). Its overall shape is most similar to those of crown-group anthropoids but it differs from the cuboids of crown anthropoids in its degree of elongation. Crown anthropoids have shorter, more nearly square-shaped cuboids in contrast to the narrower, more elongated cuboids of omomyids (Fig. 2). Despite its relative elongation, the Shanghuang cuboid differs from those of typical omomyid primates, in which the proximal part of the bone is wide and robust while that part distal to the groove for the peroneus longus tendon is mediolaterally narrow (Fig. 2). This wide (proximal) to narrow (distal) width morphology also characterizes Archicebus, a primitive tarsiiform (Ni et al., 2013). As in both adapiforms and crown anthropoids, the proximal and distal widths of the IVPP V 12319 cuboid are more nearly equal. The narrowed distal cuboid shape found among tarsiiforms likely represents a derived condition relative to other 
Eocene primate lineages. The elongation of the IVPP V 12319 cuboid suggests that it should be allocated to a Shanghuang primate taxon that also possesses some degree of distal calcaneal elongation, a feature known to occur in eosimiids. Tarsal elongation in this cuboid also suggests frequent leaping in a small primate. Given all the morphological comparisons, the Eosimiidae represents the most likely allocation for this new tarsal element, and this cuboid fits the adaptive profile noted before for eosimiids (Gebo et al., 2001). The tarsal elements of the Eosimiidae, including the cuboid described here, show morphological characteristics linking them with anthropoid primates and together they help to illustrate the initial phases of anthropoid evolution.

\section{Acknowledgments}

We thank Joshua Schwartz (Department of Geology, Northern Illinois University) for photography, Patrick Price (NIU) for artwork, and Wesley Bishop (NIU) for help with the figures. Financial support was provided by a series of grants from the National Science Foundation, including BCS 0821644 and BCS 1441585. 


\section{References}

Beard, K.C., Qi, T., Dawson, M.R., Wang, B.-Y., Li, C.-K., 1994. A diverse new primate fauna from middle Eocene fissure-fillings in southeastern China. Nature 368, 604-609.

Decker, R.L., Szalay, F.S., 1974. Origin and function of the pes in the Eocene Adapidae (Lemuriformes, Primates). In: Jenkins, F.A. (Ed), Primate Locomotion. Academic Press, New York, pp. 261-291.

Dunn, R.H., Rasmussen, D.T., 2009. Skeletal morphology of a new genus of Eocene insectivore (Mammalia, Erinaceomorpha) from Utah. J. Mam. 90, 321-331.

Fostowicz-Frelik, L., Li, C., Mao, F., Meng, J., Wang, Y., 2015. A large mimotonid from the Middle Eocene of China sheds light on the evolution of lagomorphs and their kin. Scientific Reports 5, article number 9394. doi:10.1038/srep09394

Gebo, D.L., 1987. Functional anatomy of the tarsier foot. Am. J. Phys. Anthropol. 73, 931.

Gebo, D.L., 1992. Plantigrady and foot adaptation in African apes: implications for hominid evolution. Am. J. Phys. Anthropol. 89, 29-58.

Gebo, D.L., Dagosto, M., Beard, K.C., Qi, T., 2001. Middle Eocene tarsals from China: implications for haplorhine evolution. Am. J. Phys. Anthropol. 116, 83-107.

Gebo, D.L., Dagosto, M., Ni, X.-J., Beard, K.C., 2012. Species diversity and postcranial anatomy of Shanghuang primates. Evol. Anthropol. 21, 224-238.

MacPhee, R.D.E., Beard, K.C., Qi, T., 1995. Significance of primate petrosal from middle Eocene fissure-fillings at Shanghuang, Jiangsu Province, People's Republic of China. J. Hum. Evol. 29, 501-514.

Meng, J., Bowen, G.J., Ye, J., Koch, P.L., Ting, S., Li, Q., Jin, X., 2009. Gomphos elkema (Glires, Mammalia) from the Erlian Basin: evidence for the Early Tertiarty bumbanian land mammal age in Nei-Mongol, China. Am. Mus. Nov. 3425, 1-24.

Ni, X., Gebo, D.L., Dagosto, M., Meng, J., Tafforeau, P., Flynn, J.J., Beard, K.C., 2013. The oldest known primate skeleton and early haplorhine evolution. Nature 498, 60-64.

Penkrot, T., Zack, S.P., Rose, K.D., Bloch, J.I., 2008. Postcranial morphology of Apheliscus and Haplomylus (Condylarthra, Apheliscidae): Evidence for a Paleocene 
Holarctic origin of Macroscelidea. In: Sargis, E.J., Dagosto, M. (Eds.), Mammalian Evolutionary Morphology: A Tribute to Fred Szalay. Springer Verlag, New York pp. 73-106.

Rose, K.D., Chinnery, B.J., 2004. The postcranial skeleton of early Eocene rodents. Bull. Carnegie Mus. Nat. Hist. 36, 211-244.

Salton, J.A., Szalay, F.S., 2004. The tarsal complex of Afro-Malagasy Tenrecoidea: a search for phylogenetically meaningful characters. J. Mam. Evol. 11, 73-104.

Sargis, E., 2002. Functional morphology of the hindlimb of tupaiids (Mammalia, Scandentia) and its phylogenetic implications. J. Morphol. 254, 149-185.

Szalay, F.S., 1976. Systematics of the Omomyidae (Tarsiiformes, Primates) Taxonomy, phylogeny, and adaptations. Bull. Am. Mus. Nat. Hist. 156, 159-449. 


\section{Figure Legends}

Figure 1. IVPP V 12319, a primate cuboid from Shanghuang (a, dorsal; b, medial; c, distal; d, plantar, e, lateral, and f, proximal views). Scale bar $=1 \mathrm{~mm}$.

Figure 2. Dorsal view comparisons of haplorhine cuboids (left: an omomyid, Hemiacodon; AMNH 12613, redrawn form Szalay [1976]; middle: IVPP V 12319; right: a platyrrhine, Cebus olivaceous, NIU 10-2-1). These cuboids are oriented proximal (bottom) to distal (top) with the shaded surface representing the proximal joint surface for the calcaneus. All three cuboids are drawn to approximately the same mediolateral proximal width for comparison. 
IVPP V12319

A

D

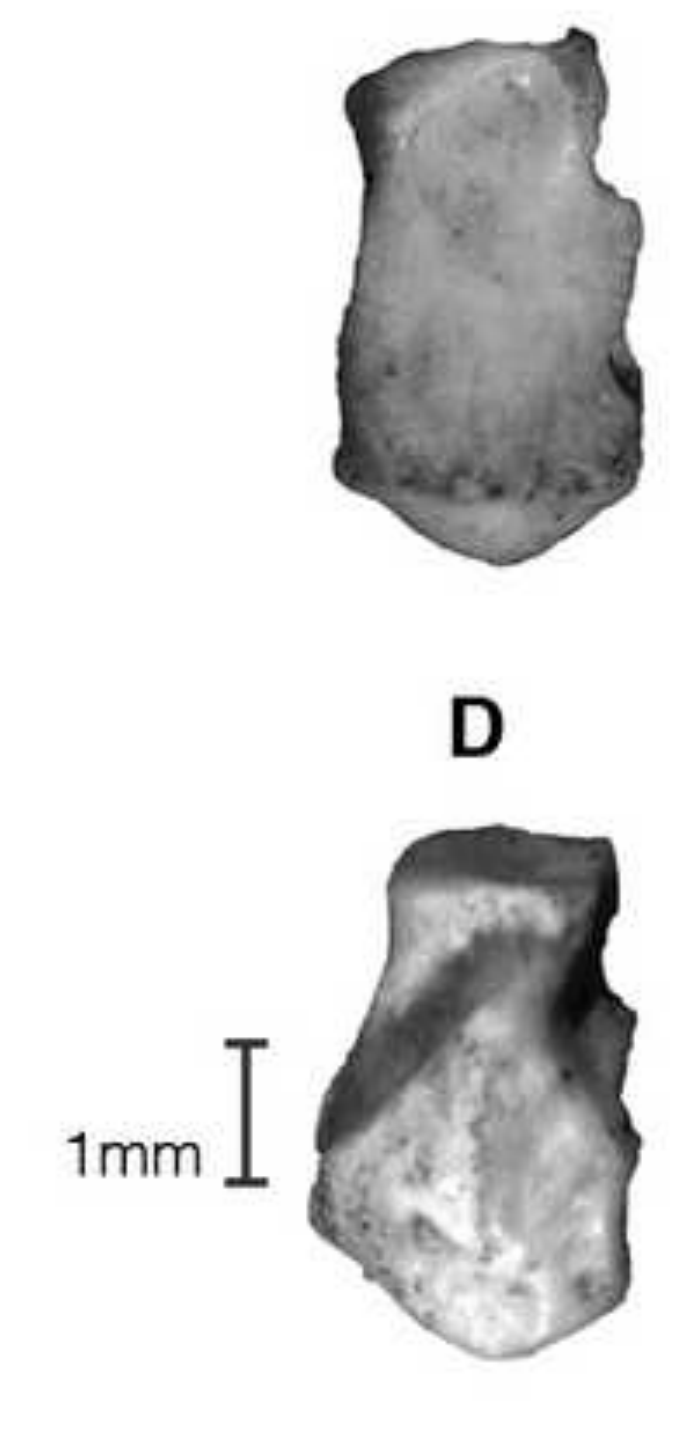

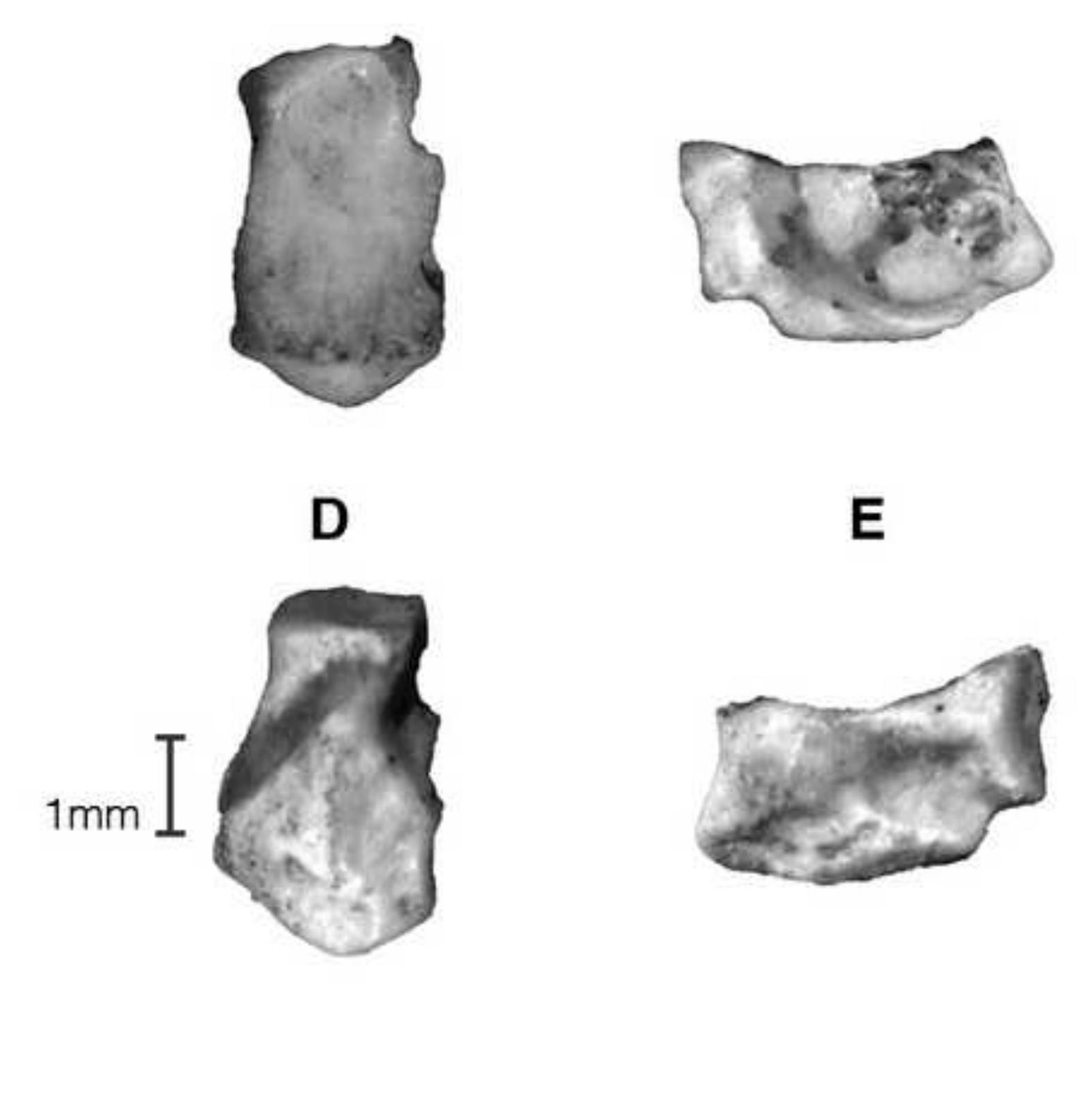

C

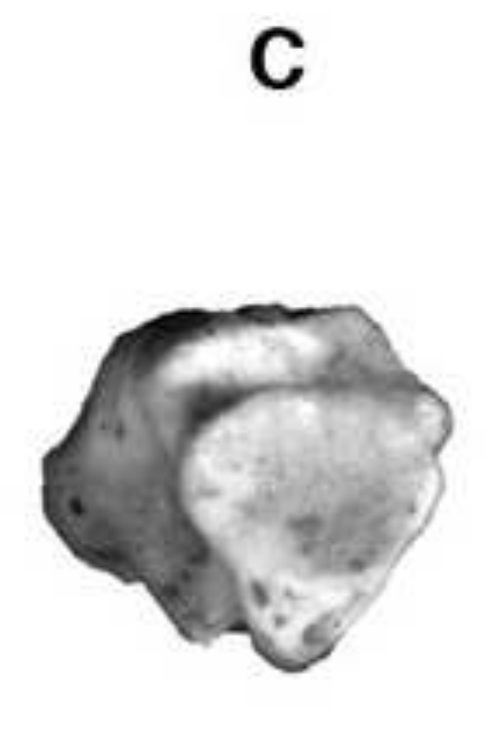

F

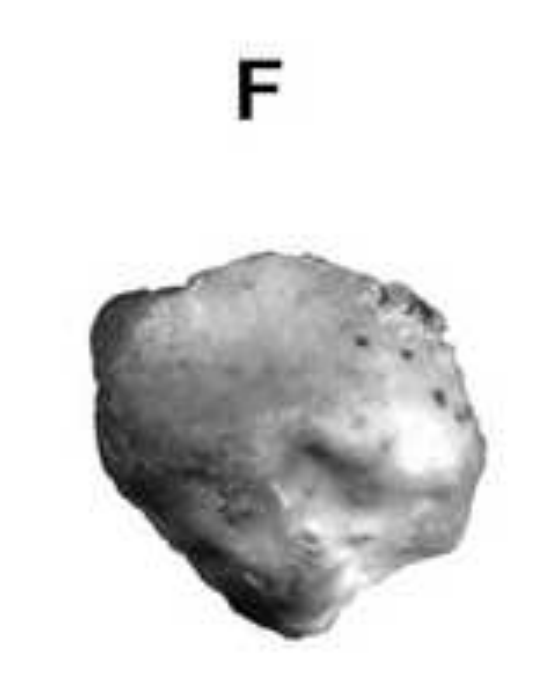$$
\text { C }
$$

$$
\text { . }
$$

.
B

E
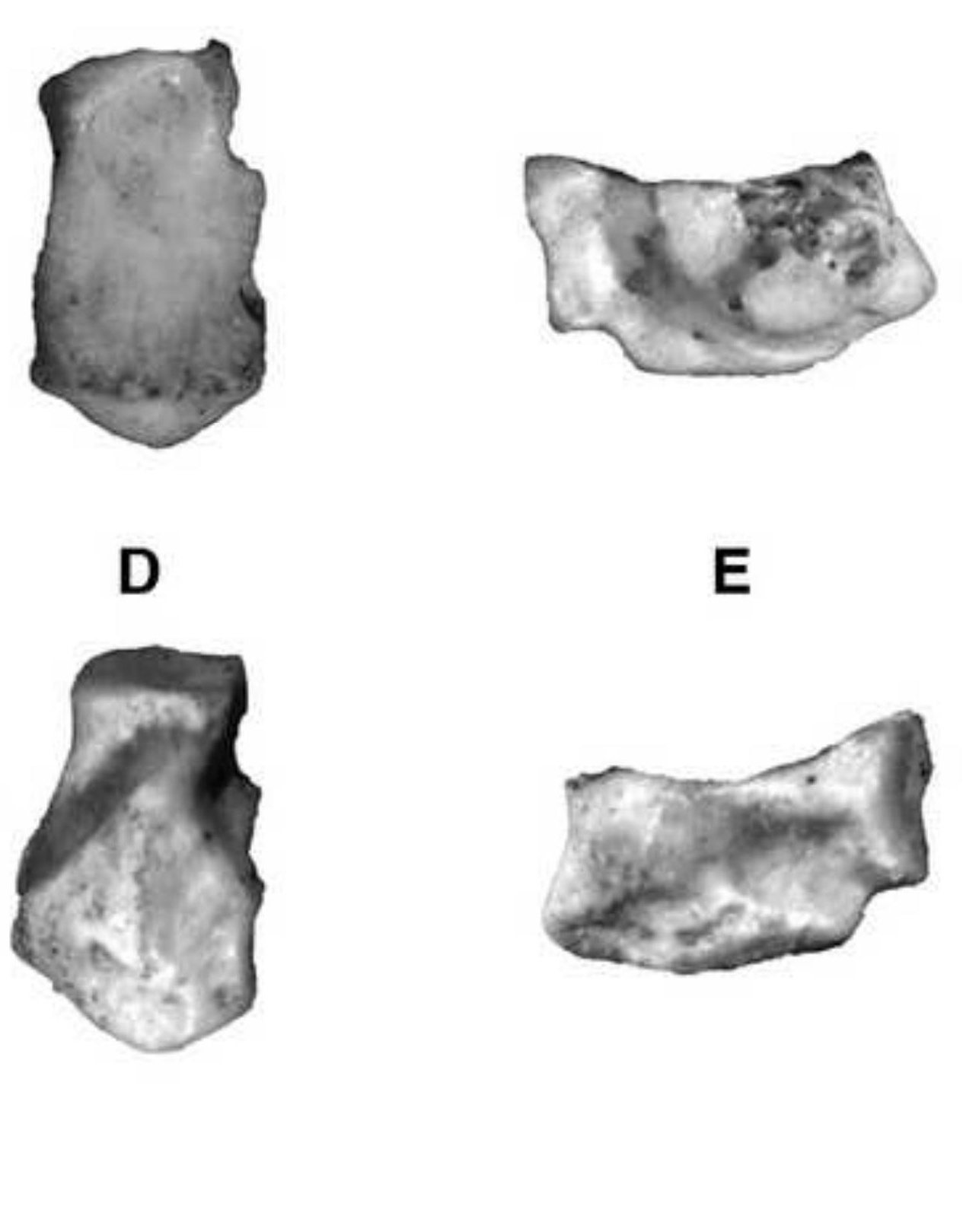
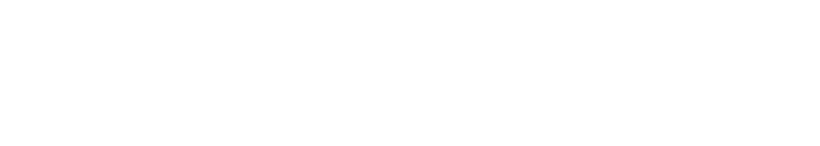

$$
\text { . }
$$
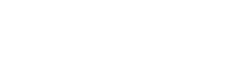


$$
B 00
$$

